\title{
ARTí́CULO
}

\section{Hongos aislados desde sedimentos de fiordos chilenos degradadores de oxitetraciclina}

\author{
Oxytetracycline degrading fungi isolated from Chilean fjord sediments
}

\author{
Ramón Ahumada-Rudolph ${ }^{1,2 *}$, Vanessa Novoa ${ }^{2}$, Anny Rudolph ${ }^{4}$ \\ Miguel Martínez ${ }^{3}$, Cristian Torres-Díaz y José Becerra ${ }^{5}$
}

\begin{abstract}
${ }^{1}$ Laboratorio de Química de Productos Naturales, Departamento de Botánica, Facultad de Ciencias Naturales y Oceanográficas, Universidad de Concepción, Casilla 160-C, Concepción, Chile. *ramonahumada @udec.cl

${ }^{2}$ Estudiantes de Doctorado en Ciencias Ambientales, Facultad de Ciencias Ambientales, Universidad de Concepción, Casilla 160-C, Concepción, Chile

${ }^{3}$ Laboratorio de Microbiología Básica y Bioremediación, Facultad de Ciencias Biológicas, Universidad de Concepción, Víctor Lamas 1290, Ciudad Universitaria, Concepción, Chile

${ }^{4}$ Facultad de Ciencias, Universidad Católica de la Santísima Concepción, Alonso de Ribera 2850, Concepción, Chile

${ }^{5}$ Laboratorio de Genómica \& Biodiversidad (LGB), Departamento de Ciencias Naturales, Universidad del Biobío, Dieciocho de Septiembre 580, Chillán, Chile
\end{abstract}

\begin{abstract}
It is proposed that marine fungi are a good alternative for remediation of areas contaminated with antibiotics due to their heterotrophic condition, adaptability to environmental changes, and specific resistance to stressors. With this assumption we investigated the fjords of Southern Chile, which are characterized by salmon farming. We evaluated the concentration of oxytetracycline (OTC) in sediments from different areas surrounding salmon cages. In addition, we isolated fungi species to assess their ability to degrade OTC. Measurable amounts of OTC were detected in deeper sediments located at the eastern sector of cages and rafts $\left(Z=100 \mathrm{~m} ; 4.62 \pm 0.12\right.$ to $24.1 \pm 1.03 \mu \mathrm{g} \mathrm{g} \mathrm{g}^{-1}$. Thirty-eight strains of endemic fungi were isolated and identified in the sediments near the farming center. Among these strains we detected species from the Phylum Ascomycetes, including Penicillium commune, Aspergillus terreus, Beauveria bassiana, Trichoderma harzianum, Epicoccum nigrum and Emericellopsis alkaline. These strains fungi were capable of degrading about 76 to $92 \%$ of the OTC present in the culture media. The results of this study constitute a first step into implementing processes that could favor waste bioremediation from marine sediments.
\end{abstract}

Key words: Degradation, marine fungi, Ascomycota, oxytetracycline, bioremediation

Resumen.- Los hongos marinos por su condición heterótrofa, su adaptabilidad a la variabilidad ambiental y su resistencia específica a diferentes estresores, son una opción para ser empleados como restauradores de sectores contaminados con antibióticos. Con esta hipótesis se realizó la investigación en una zona de cultivo de salmones en el sur de Chile. Para ello se evaluó la concentración de oxitetraciclina (OTC) de sedimentos de diferentes zonas cercanas a jaulas de cultivo de salmón. También se aisló de sedimentos cepas de hongos para evaluar su capacidad de degradar OTC. Los resultados obtenidos mostraron en el área de estudio concentraciones de OTC que fluctuaron entre 4,62 $\pm 0,12$ a $24,1 \pm 1,03 \mu \mathrm{g} \mathrm{g}^{-1}$, en el sector Este a la balsa jaula y en los sedimentos de $100 \mathrm{~m}$ de profundidad. Además, de estos sedimentos se aisló 38 cepas de hongos y se identificó y trabajó con 6 cepas del Phylum Ascomycetes: Penicillium commune, Aspergillus terreus, Beauveria bassiana, Trichoderma harzianum, Epicoccum nigrum y Emericellopsis alkalina. Las pruebas de biodegradación demostraron que las cepas fueron capaces de degradar OTC en porcentajes que fluctuaron entre 76 y 92\%. Los resultados representan un primer paso en la implementación de procesos que permitan la biorremediación de residuos de OTC desde los sedimentos.

Palabras clave: Degradación, hongo marino, Ascomycota, oxitetraciclina, biorremediación

\section{INTRODUCCIÓN}

La oxitetraciclina (OTC) es un antibiótico de amplio espectro incluido en el grupo de las tetraciclina y uno de los dos antibióticos (junto a la estreptomicina) registrados por la EPA para uso veterinario, como tratamiento profiláctico. La OTC es de bajo costo y alta eficiencia, debido a que interfiere en la síntesis de proteínas bacterianas (Vidaver 2002). Se utiliza en una amplia variedad de actividades, acuicultura, ingeniería genética, experimentos de investigación, crianza de juveniles y producción de cultivos (Dietze et al. 2005).

En la salmonicultura antibióticos como OTC son administrados en conjunto con la dieta alimentaria, tanto en la fase de alevinaje en agua dulce, como en el proceso de engorda de salmones en sectores marinos. Para el 2013 se estima que 
se aplicaron alrededor de 157 ton de OTC a los sistemas de cultivo; el 92\% de su uso se justificó por la enfermedad de piscirickettsiosis (SERNAPESCA 2014). La OTC presenta alta solubilidad en agua y es poco absorbida en el intestino del animal (Alcock et al. 1999). La literatura indica que un $80 \%$ de la dosis administrada de OTC, podría ser eliminada por la orina y heces de los animales en la fase de engorda (Hektoen et al. 1995).

El impacto de la aplicación de OTC tiene directa relación con la dosis, concentración y tiempo de aplicación (Cabello 2004, 2006; Buschmann et al. 2006, 2012, 2016). Su efecto abarca un amplio espectro de bacterias, no sólo las patógenas. Además, se ha identificado presencia de poblaciones de bacterias resistentes a oxitetraciclina en la microflora de granjas salmoneras (Cabello et al. 2013), las cuales serían reservorios de bacterias portadoras de determinantes genéticos transferibles de resistencia a antibióticos, lo que constituye un riesgo para la salud (Miranda \& Zemelman 2002, Heuer et al. 2009, Marshall \& Levy 2011, Bannister et al. 2014, Andrade et al 2015).

En la Región de Aysén, existen alrededor de 635 concesiones para cultivos de salmónidos, siendo ésta la principal actividad económica en el área. En el 2014 se reportó una producción de 850 mil toneladas (SUBPESCA 2014 ${ }^{1}$, Buschmann \& Muñoz 2016). La salmonicultura es una actividad que genera problema ambientales en el borde costero por el ingreso de antibióticos, altos contenidos de materia orgánica, nutrientes, disminución en el contenido de oxígeno disuelto, acidificación del sedimento y alteraciones ecológicas (Rudolph et al. 2009, 2011). En la actualidad los planes de mitigación de los centros de cultivo aún consisten en sólo su relocalización (SUBPESCA 2015) ${ }^{2}$, en espera que con el transcurso del tiempo mejore la calidad de los sedimentos en las áreas intervenidas ambientalmente.

Los hongos marinos no han sido suficientemente estudiados como degradadores de xenobióticos (Williams et al. 2007). En relación a los costos de la micorremediación para remover contaminantes, se estima que es menos costosa que los métodos físico químicos equivalentes. Por ejemplo, la micorremediación no requiere de realizar excavaciones para tratar el suelo y agua en el subsuelo, permitiendo conservar la estructura del sustrato en que se aplica, reduciendo el impacto sobre los sistemas naturales (Magan et al. 2010).

En este estudio la hipótesis de trabajo es que los hongos marinos por su condición heterótrofa, su adaptabilidad a la variabilidad ambiental y su resistencia específica a diferentes estresores, son una opción a analizar para ser empleados como degradadores de antibióticos. El objetivo de este trabajo fue aislar e identificar cepas de hongos endémicos, desde sedimentos cercanos a balsas jaulas de cultivo de salmónidos y determinar su capacidad para degradar el antibiótico OTC.

\section{MATERIALES Y MÉTODOS}

\section{RECOLECCIÓN DE SEDIMENTOS}

Los sedimentos fueron recolectados en julio de 2013, desde fiordos de la provincia de Aysén (45¹2'35,33''S $74^{\circ} 04^{\prime} 25^{\prime \prime} \mathrm{W}$ ) (Fig. 1). Para ello se utilizó una embarcación menor y una draga de acero inoxidable de $1.800 \mathrm{~cm}^{3}$ de capacidad. Se recolectaron 16 muestras (con 3 réplicas cada una) desde los primeros $5 \mathrm{~cm}$ de sedimento, en puntos equidistantes en torno a las balsas de cultivo, a una distancia aproximada de $5 \mathrm{~m}$. Cada muestra y sus réplicas fueron almacenadas a $-18^{\circ} \mathrm{C}$ en tubos Falcon esterilizados en el centro de cultivo, para luego ser trasladadas refrigeradas hasta el laboratorio, donde finalmente se almacenaron a $-18^{\circ} \mathrm{C}$.

\section{Cuantificación de OTC en Sedimento}

De cada muestra se tomó $3 \mathrm{~g}$ de sedimento húmedo, los cuales fueron mezclados y homogeneizados en $30 \mathrm{~mL}$ de ácido oxálico 0,01 M en metanol. Se aplicó ultrasonido con un equipo Branson modelo 291 de $40 \mathrm{kHz}$ por 15 min y centrifugó a 4.000 x g por $20 \mathrm{~min}$. El sobrenadante fue recuperado, trasvasijado y secado a presión reducida a $50^{\circ} \mathrm{C}$. Se reconstituyó la muestra en $3 \mathrm{~mL}$ con agua:acetonitrilo en proporción 75:25 y filtró con PVDF $0,22 \mu \mathrm{m}$ para luego inyectar en HPLC-DAD. Se preparó una curva de calibrado entre 0 a $100 \mu \mathrm{g} \mathrm{g}^{-1}$, con sedimento enriquecido con OTC para la cuantificación.

\section{Aislamiento e identificación de las cepas}

De cada muestra se tomó $3 \mathrm{~g}$ de sedimento y se diluyó en agua de mar estéril, homogeneizó y se dejó decantar por $15 \mathrm{~min}$. Se realizó una dilución seriada con agua de mar esterilizada, agregando $1 \mathrm{~mL}$ en $9 \mathrm{~mL}$ de agua de mar, con 2 repeticiones. Se tomó una alícuota de $100 \mu \mathrm{L}$ que fue diseminada en la superficie de una placa con agar YMG (levadura, malta y glucosa), con adición de estreptomicina para evitar el desarrollo de bacterias (200 $\left.\mathrm{mg} \mathrm{L}^{-1}\right)$ y se incubó a temperatura ambiente durante 5 días para luego aislar las cepas.

${ }^{1}$ SUBPESCA. 2014. Informe sectorial de pesca y acuicultura. Subsecretaría de Pesca y Acuicultura. Valparaíso, Chile <http:// Www.subpesca.cl/publicaciones/606/articles-86801_documento.pdf>

${ }^{2}$ SUBPESCA. 2015. Subsecretaría de Pesca y Acuicultura. Valparaíso, Chile <http://www.subpesca.cl/prensa/601/w3-article-88821.html> 
Figura 1. Sitio de muestreo. Estación 'Centro de cultivo Victoria 1', campaña de invierno 2013. Ubicación vecina a las concesiones marítimas para el cultivo de salmones (según SERNAPESCA 2014) / Sampling site. 'Victoria 1 culture center' Station, Winter Champaign. 2013. Location neighboring maritime concessions for salmon farming in the area, as described by SERNAPESCA 2014

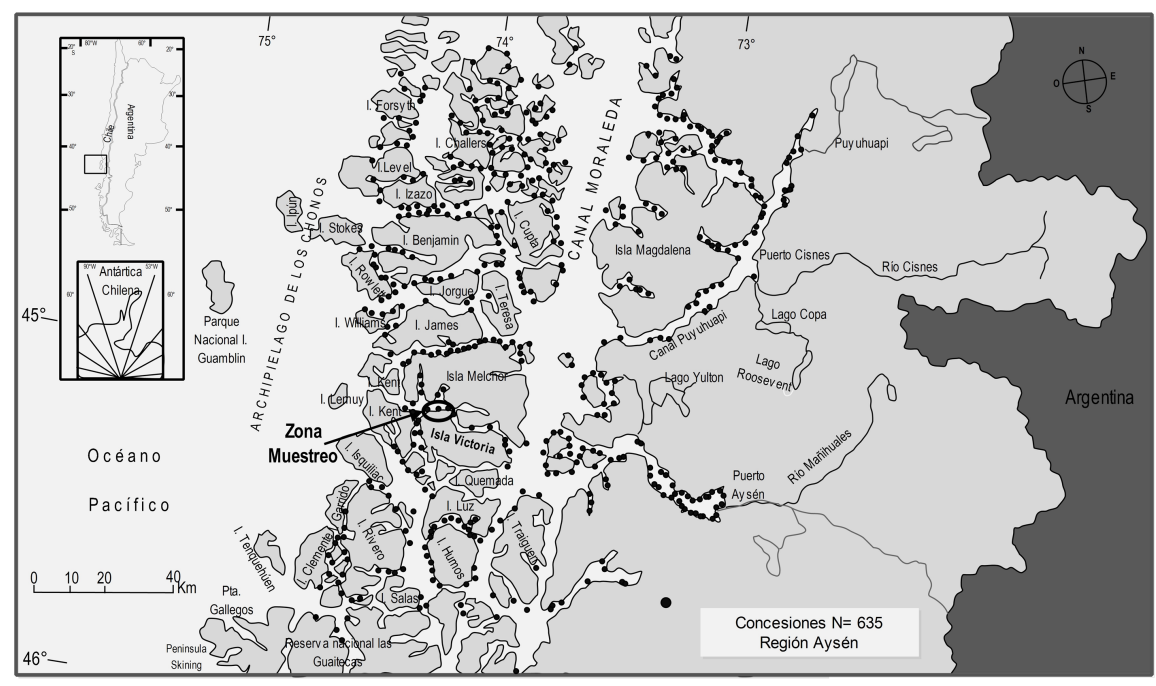

La determinación y caracterización morfológica de las cepas, se realizó utilizando microscopía electrónica de barrido. Para la validación de las especies, se utilizó técnicas de PCR. Para ello se amplificó la región ITS (que incluye el gen 5.8S) y las subunidades nucleares ribosomales de rRNA (LSU) utilizando ITS-1/ITS-4 (Tedersoo et al. 2009). Se extrajo el ADN del micelio utilizando el MiniKit de extracción de ADN fúngico EZNA (Omega-Biotek). Las reacciones de PCR se realizaron con ITS4 (5'- tcctccgcttattgatatgc-3') como partidores directos y ITS1 (5'-tccgtaggtgaacctgcgg-3') como partidores inversos. Para cada reacción se utilizó $15 \mu \mathrm{L}$ de solución que contenía 30-50 ng de ADN, $1 X$ de tampón de PCR, 2 mM MgCl $2,0,1$ $\mathrm{mM}$ de cada dNTP, $0,5 \mathrm{mM}$ de los cebadores directos e inverso, y $1 \mathrm{U}$ de Taq ADN polimerasa. Las amplificaciones por PCR se llevaron a cabo con una desnaturalización inicial de 4 min a $94^{\circ} \mathrm{C}$, seguido de 35 ciclos de $30 \mathrm{~s}$ a $94^{\circ} \mathrm{C}, 60 \mathrm{~s}$ a $50^{\circ} \mathrm{C}, 60 \mathrm{~s} \mathrm{a} 72^{\circ} \mathrm{C}$ y un paso final de $5 \mathrm{~min}$ a $72^{\circ} \mathrm{C}$. El producto de PCR se purificó y secuenció en ambos sentidos. Secuenciadas las cepas fueron editadas utilizando Genious software v5.4 (Drummond et al. 2011). La secuencia se analizó con BLAST (Basic Local Alignment Search Tool) (http:// blast.ncbi.nlm.nih.gov/Blast.cgi), para la determinación del porcentaje de identidad máxima se utilizó las secuencias de la base mundial de datos. Las secuencias obtenidas fueron depositadas en la GenBank ${ }^{\circledR}$. Cada una de las cepas aisladas en este trabajo, fueron almacenadas en el Cepario del Laboratorio de Química de Productos Naturales de la Universidad de Concepción.

\section{Evaluación de degradación de OTC}

Se cultivó las esporas de cada cepa por 4 días en 125 ml YMG líquido. Se tomó $5 \mathrm{~g}$ de micelio de cada cepa los que fueron cultivados en matraz Erlenmeyer de $250 \mathrm{~mL}$ con $125 \mathrm{~mL}$ de solución con $250 \mathrm{mg} \mathrm{L}^{-1}$ de oxitetraciclina. Cada cepa y sus respectivos controles fueron trabajados en triplicado, a temperatura ambiente, oscuridad y agitación constante $(120 \mathrm{x}$ g). Como control negativo se utilizó solución de oxitetraciclina a una concentración de $250 \mathrm{mg} \mathrm{L}^{-1}$. Los ensayos fueron controlados a los $0,3,6,9,12,15$ y 18 días de cultivo. Para ello, una alícuota de $1 \mathrm{~mL}$ de cada ensayo y controles fue filtrada en filtros de 0,23 $\mu \mathrm{m}$ de poro e inyectada en HPLC-DAD, para evaluar su actividad degradadora de oxitetraciclina. A partir de un estándar de oxytetracycline hydrocloride al 98,9\% pureza, CAT\# 500105 Calbiochem, se preparó una curva de calibración con $7,15,30,100,175$ y $250 \mathrm{mg} \mathrm{L}^{-1}$. Características de la curva: $y=25,89 x-115,1 ; R^{2}=0,998$; error típico $=1,59 \mathrm{mg} \mathrm{L}^{-1}$; con límites de detección y cuantificación de 6,03 y $6,46 \mathrm{mgL}^{-1}$, respectivamente.

\section{AnÁlisis estadísticos}

Se realizó un análisis de varianza de medidas repetidas, que consideró 2 factores: Tipo de cepa y tiempo de exposición en días. Además, se aplicó la prueba a posteriori de Tukey y se utilizó el programa Statistica v. 8.0.

\section{Resultados}

En los análisis de sedimentos en sectores aledaños a las balsas de engorda de salmónidos, se detectó cantidades medibles de OTC en 3 de las 16 muestras. Específicamente en el sector Este a la balsa y en los sedimentos con profundidades de: $5 \mathrm{~m}$ $\left(4,62 \pm 0,12 \mu \mathrm{g} \mathrm{g}^{-1}\right), 15 \mathrm{~m}\left(5,3 \pm 0,10 \mu \mathrm{g} \mathrm{g}^{-1}\right)$ y $100 \mathrm{~m}(24,1 \pm$ $\left.1,03 \mu \mathrm{g} \mathrm{g}^{-1}\right)$ de profundidad. 
De las 38 cepas aisladas desde los sedimentos del área de fiordos de la provincia de Aysén, se seleccionaron 6 cepas por su capacidad de degradar OTC. En la Tabla 1 se indican las cepas estudiadas, filo, orden y código Genbank ${ }^{\circledR}$. En la Figura
2 se muestras aspectos morfológicos obtenidos por microscopía electrónica de barrido los que fueron utilizados, en una primera aproximación, para su identificación.

Tabla 1. Nombre y códigos de acceso al Genbank ®de las cepas con capacidad para degradar OTC identificadas en este estudio / Name and GenBank ${ }^{\circledR}$ access codes for strains with the ability to degrade OTC identified in the study

\begin{tabular}{llcll}
\hline Cepa & \multicolumn{1}{c}{ Nombre científico } & Filo & Orden & Cód. Genbank $^{\circledR}$ \\
\hline RA117 & Penicillium commune & Ascomycota & Eurotiales & KU298470 \\
RA106 & Aspergillus terreus & Ascomycota & Eurotiales & KU214241 \\
RA129 & Beauveria bassiana & Ascomycota & Hypocreales & KU198598 \\
RA115 & Trichoderma harzianum & Ascomycota & Hypocreales & KU198601 \\
RA39P & Epicoccum nigrum & Ascomycota & Pleosporales & KC164754 \\
RA2P & Emericellopsis alkalina & Ascomycota & Hypocreales & KU298468 \\
\hline
\end{tabular}

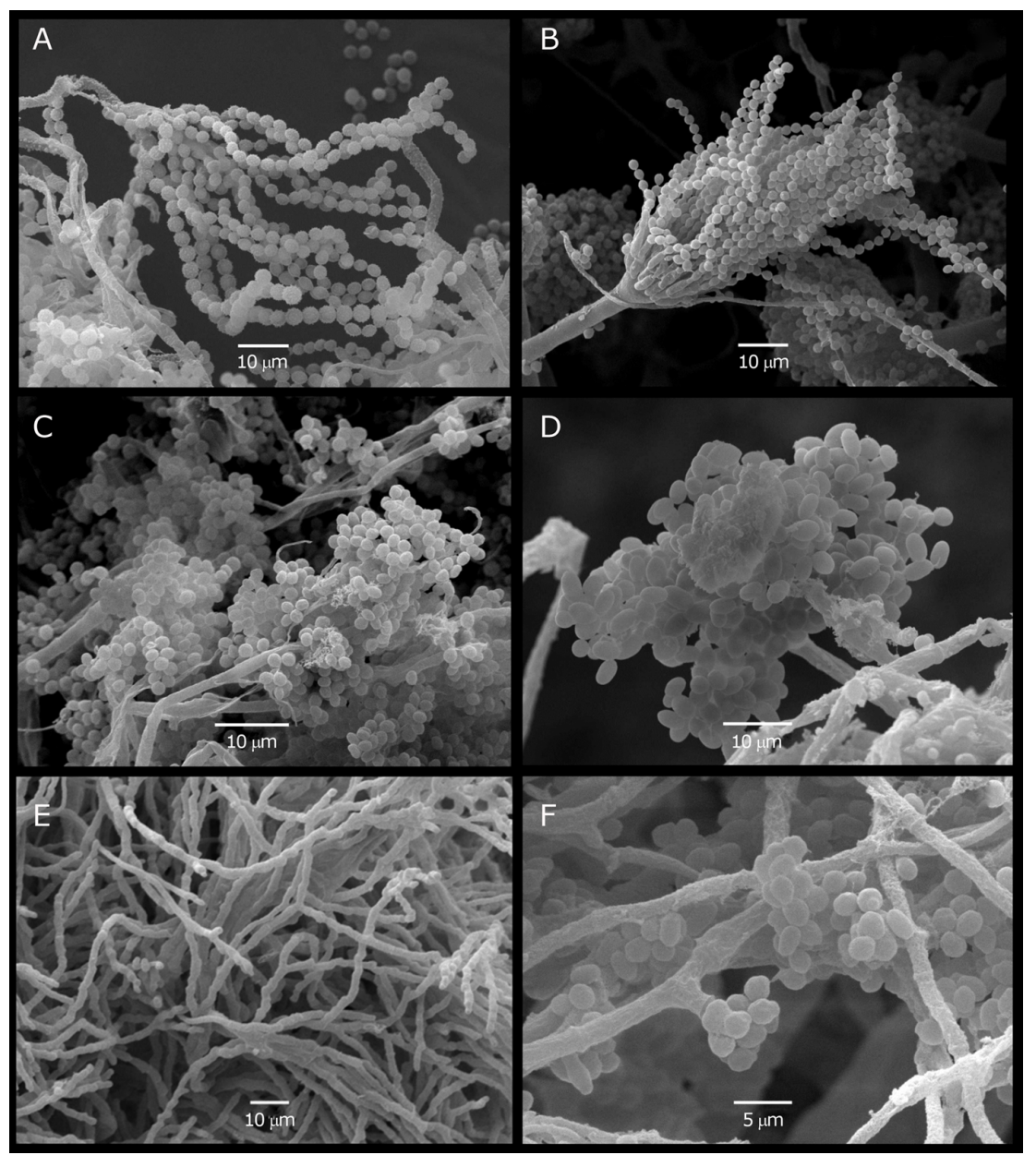

Figura 2. Fotografía de microscopía electrónica de barrido de cepas aisladas e identificadas desde sedimentos marinos, con capacidad para degradar OTC: A) P. commune, B) A. terreus, C) B. bassiana, D) T. harzianum, E) E. nigrum y F) E. alkalina / Photographs of strains with the ability to degrade OTC isolated and typified from marine sediments: A) P. commune B) A. terreus C) B. bassiana D) T. harzianum E) E. nigrum and F) E. alkaline. Images obtained by scanning electron microscopy 


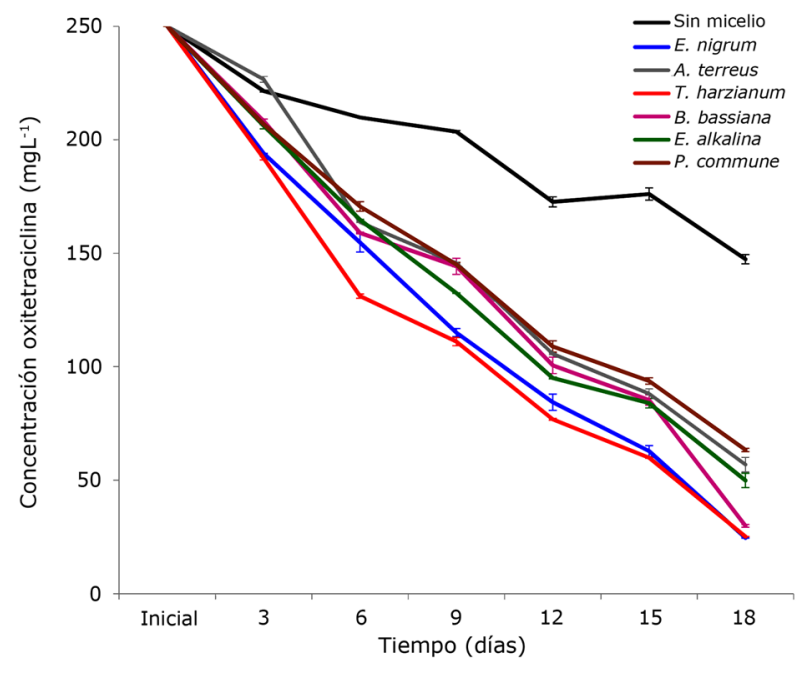

Figura 3. Degradación de OTC de las diferentes cepas aisladas desde sedimentos, determinados por HPLC-DAD. Las barras representan el error estándar (e.e)/ Effects of OTC concentration on culture media for different marine strains and times of exposure as determined by HPLC-DAD. Bars represent the standard error (s.e)

Los ensayos de degradación de OTC demostraron que las 6 especies de hongos presentaron diferencias significativas de degradación respecto al control (Fig. 3). Al finalizar del tiempo de exposición de OTC, las cepas que presentaron mejor eficiencia de degradación de mayor a menor fueron: Epicoccum nigrum (20,6 $\left.\mathrm{mg} \mathrm{L}^{-1}\right)$; Trichoderma harzianum (21,3 $\left.\mathrm{mg} \mathrm{L}^{-1}\right)$ y Beauveria bassiana $\left(26,1 \mathrm{mg} \mathrm{L}^{-1}\right)$, seguido de Emericellopsis alkaline $\left(46,7 \mathrm{mg} \mathrm{L}^{-1}\right)$. Menores eficiencias se observaron en: Aspergillus terreus $\left(53,9 \mathrm{mg} \mathrm{L}^{-1}\right)$ y Penicillium commune (60,5 mg L $\mathrm{m}^{-1}$ ) (Fig. 4). El control (cultivos sin micelio y con OTC) presentó $147 \mathrm{mg} \mathrm{L}^{-1}$ de degradación, correspondiente a la degradación natural de OTC en condiciones de laboratorio.

\section{Discusión}

La rápida expansión de la salmonicultura i.e., 635 concesiones, sólo en la Región de Aysén (Fig. 1) y la necesidad de mitigar el efecto adverso de la incorporación de antibióticos en el ambiente, incentivó la búsqueda de organismos capaces de aumentar la velocidad de degradación de antibióticos, como la OTC, comúnmente usado en los centros de cultivo. Antecedentes bibliográficos plantean que la velocidad de degradación de la OTC en condiciones naturales en los sedimentos marinos es baja (Samuelsen et al. 1994) y que su vida media sería función de la tasa de reingreso de la oxitetraciclina hacia la columna de agua (Smith \& Samuelsen 1996).
En este estudio, la mayor concentración medida de OTC en los sedimentos en torno a la balsa jaula, fue de $24,1 \pm 1,03 \mu \mathrm{g}$ $\mathrm{g}^{-1}$, mayor al rango de concentraciones informada para 13 centros de salmónidos, i.e., 0,1 y $11 \mu \mathrm{g} \mathrm{g}^{-1}$ (Bjorklund et al. 1991, Coyne et al. 1994, Kerry et al. 1995), pero inferior a

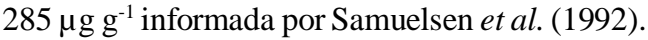

Para la determinación de la capacidad de adsorción de xenobióticos a una matriz ambiental, se trabaja con la constante de adsorción $(\mathrm{kd})$, que relaciona la concentración del compuesto en la fase de adsorción y el agua con que se encuentra en equilibrio. En sedimentos orgánicos marinos se informa $\mathrm{Kd}$ para la OTC de $2.590\left(\mathrm{~L} \mathrm{~kg}^{-1}\right)$, superior al informado para sustratos como fangos arenosos de sistemas agrícolas, con alrededor de $670\left(\mathrm{~L} \mathrm{~kg}^{-1}\right)$ (WingChing-Jones 2008), lo que podría explicar las altas concentraciones de OTC en sedimentos marinos respecto de suelos agrícolas. Por otra parte, la concentración de OTC en los sedimentos en el área de la balsa monitoreada, fueron mayores en el sector Este y a mayor profundidad i.e., $100 \mathrm{~m}$, lo que indicaría una relación entre las corrientes, el transporte de sedimento y la concentración de OTC (Smith 1996). Además, Hektoen et al. (1995), plantearon que a mayor profundidad de la columna de agua y mayores contenido de materia orgánica, el tiempo de vida media de la OTC sería mayor de 100 días, lo que generaría acumulación de OTC en sedimentos de mayor profundidad en directa relación con el contenido de materia orgánica del sedimento.

Los microorganismos son la fuerza motriz de los ciclos biogeoquímicos y el puente que une los procesos entre los ecosistemas (Kong et al. 2011). El mantenimiento de la actividad biológica es clave para garantizar el funcionamiento de los ecosistemas, debido a ello, la acumulación de antibióticos es una amenaza potencial a las funciones y estructura de las comunidades bacterianas (Liu et al. 2012, Seo et al. 2009) pero, no para comunidades de hongos, siendo esta una clara ventaja para su utilización en la micoremediación de antibióticos. Ejemplos exitosos de la utilización de hongos en matrices complejas, es la aplicación de basidiomicetos, especialmente sub Filo Agaricomycetes, en la degradación y tratamiento de emisarios; de hongos asociados a la degradación de la madera como: Pleorotus ostreatus, Irpex lacteus, Trametes versicolor, Phanerocheate chrysosporium, y Bjerkandera adusta; y los que crecen en bosques de coníferas como: Lentinula edodes, Agaricus bisporus y Lactarius piperatus, capaces de degradar compuestos refractarios como celulosa, lignina, residuos de la fabricación de papel, plásticos, hidrocarburos, colorantes, pesticidas, productos farmacéuticos (Anastasi et al. 2013, Kulshreshtha et al. 2014). 


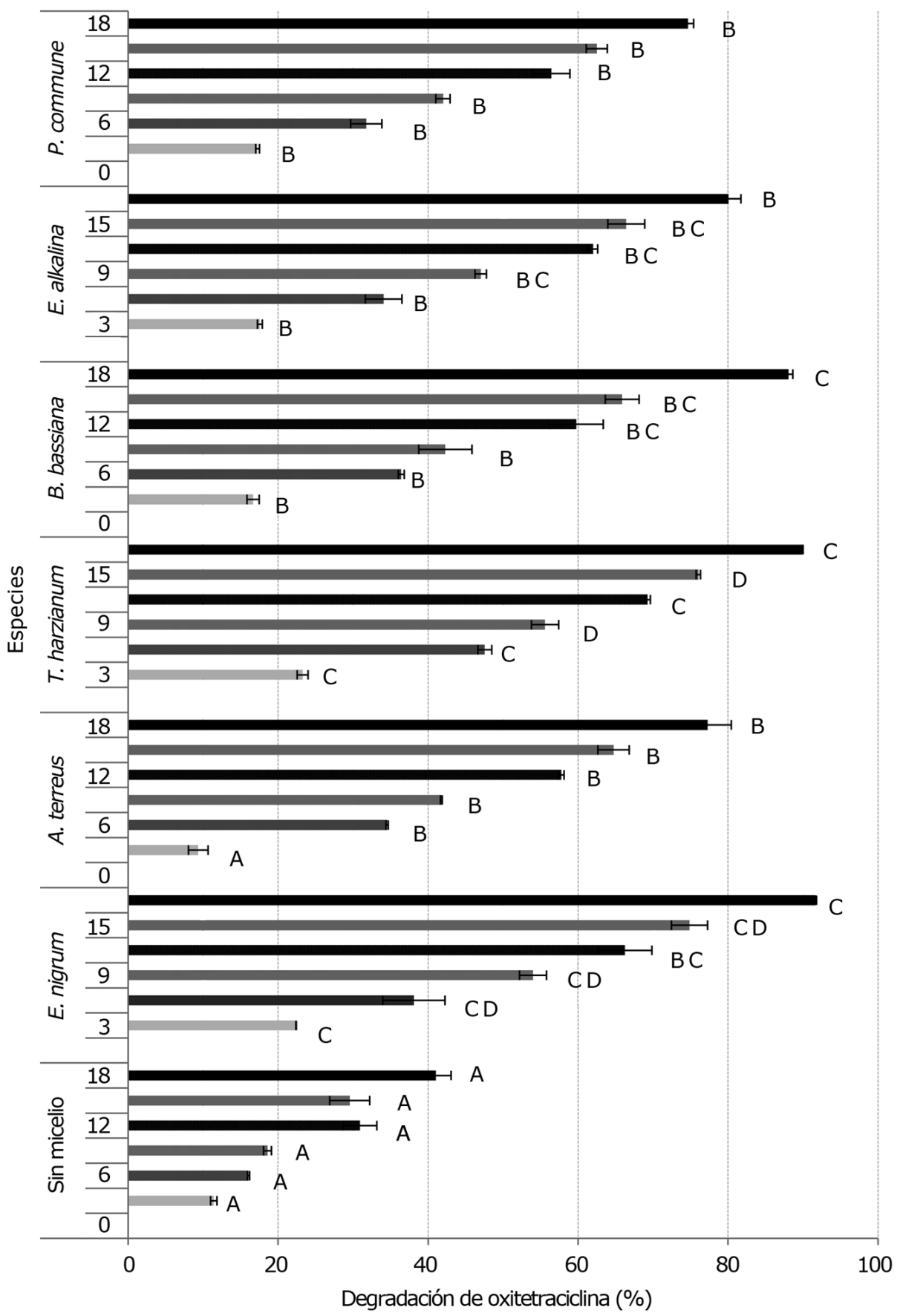

Figura 4. El comportamiento de degradación de OTC de cada cepa, en los tiempos de medición: 0, 2, 6, 10, 15 y 18 días. $(*)$ Medias con letra común no son significativamente diferentes $(P>0,05) /$ The degradative behavior of OTC of each strain, in the measured times: $0,3,6,10,15$ and 18 days. $\left({ }^{*}\right)$ Measurements with the same letter are not significantly different $(P>0.05)$ 
Kristanti \& Hadibarata (2015) plantean la potencialidad de lograr mayores eficiencias si se trabajaba aislando hongos endémicos (por Ej. filo Ascomycota), de sitios contaminados con las sustancias que se desea degradar. Esto se prueba en este estudio, ya que se observó que las 6 cepas aisladas desde los sedimentos y expuestas a OTC, mostraron capacidad para degradar este antibiótico (76 a 92\%) y ser capaces de sobrevivir y crecer en presencia de una cantidad muy superior del antibiótico en condiciones de laboratorio $\left(250 \mu \mathrm{g} \mathrm{L}^{-1}\right)$, respecto a la concentración más alta en que vivían en el sustrato del que fueron aisladas $\left(24,1 \pm 1,03 \mu \mathrm{g} \mathrm{g}^{-1}\right)$. Otras ventajas de utilizar hongos en procesos de biorremediación es que los hongos son capaces de formar extensas redes de micelio, llegando a ser la biomasa dominante y además, no necesitan como pre-condición el enriquecimiento del sustrato, como sucede con muchas bacterias (Maloney 2001, Magan et al. 2010, Harms et al. 2011).

En estudios previos, trabajando en 21 días, se observó baja capacidad degradativa de OTC de la levadura $R$. mucilaginosa y resultados promisorias con 4 cepas de ascomicetes aisladas sede sedimentos marinos; sus capacidades degradativas que fluctuaron entre 72 y $92 \%$ (Ahumada-Rudolph et al. 2016). En este estudio una de las cepas con mayor eficiencia para degradar OTC fue E. nigrum (91,7\% de eficiencia). En estudios previos con esta cepa se habían demostrado en sus paredes celulares diferencias significativas en la composición de esteroles y ácidos grasos, característica que podrían ser responsables de su capacidad adaptativa para co-habitar en sustratos marinos y terrestres, siendo por ello un excelente candidato para tratamientos de micorremediación en los centros de reproducción y crecimiento (smolt) en agua dulce, como en centros marinos de engorda (Ahumada-Rudolph et al. 2014).

En este contexto, los resultados demuestran que los hongos marinos aislados desde fiordos pueden ser una buena alternativa para usarlos como potenciales degradadores de OTC y restaurar sectores contaminados con éstos antibióticos.

\section{Agradecimientos}

Los autores agradecen el soporte financiero al proyecto 'CONICYT + PAI Concurso Nacional Tesis de Doctorado en la Industria', Convocatoria 2013 Folio 783110001, a la empresa asociada al Proyecto Blumar Salmones S.A., al proyecto FONDECYT 1151028 y a la Vicerrectoría de Investigación y Desarrollo de la Universidad de Concepción. Al Sr. Nicos Nicolaides por el apoyo a esta investigación. Además agradecemos los comentarios de los evaluadores cuyas sugerencias fueron un aporte a mejorar el manuscrito.

\section{LiTERATURA CITADA}

Ahumada-Rudolph R, D Cajas-Madriaga, A Rudolph, R Reinoso, C Torres, M Silva \& J Becerra. 2014. Variation of sterols and fatty acids as an adaptive response to changes in temperature, salinity and $\mathrm{pH}$ of a marine fungus Epicoccum nigrum isolated from the Patagonian Fjords. Revista Biología Marina y Oceanografía 49(2): 293-305.

Ahumada-Rudolph R, V Novoa, K Sáez, M Martínez, A Rudolph, C Torres-Diaz, J Becerra. 2016. Marine fungi isolated from Chilean fjord sediments can degrade oxytetracycline. Environmental Monitoring and Assessment. <doi: 10.1007/s10661-016-5475-0>

Alcock RE, A Sweetman \& KC Jones. 1999. Assessment of organic contaminant fate in wastewater treatment plants I. Selected compounds and physiochemical properties. Chemosphere 38: 2247-2262.

Anastasi A, V Tigini \& GC Varese. 2013. The bioremediation potential of different ecophysiological group of fungi. In: Goltapeh EM, YR Danesh \& A Varma (eds). Fungi as bioremediators, pp. 479-489. Springer-Verlag Berlin Heidelberg.

Andrade VdaC, BdelB Zampieri, ER Ballesteros, AB Pinto \& AJ de Oliveira. 2015. Densities and antimicrobial resistance of Escherichia coli isolated from marine waters and beach sands. Environmental Monitoring and Assessment 187: 342. <doi: 10.1007/s10661-015-4573-8>

Bannister RJ, T Valdemarsen, PK Hansen, M Holmer \& A Ervik. 2014. Changes in benthic sediment conditions under an Atlantic salmon farm at a deep, well-flushed coastal site. Aquaculture Environment Interactions 5: 29-47.

Bjorklund HV, CMI Rlbergh \& G Bylund. 1991. Residues of oxolinic acid and oxytetracycline in fish and sediments from fish farms. Aquaculture 97: 85-96.

Buschmann AH \& JLP Muñoz. 2016. Salmonid farming. Reference Module in Earth Systems and Environmental Sciences, Elsevier. <doi: 10.1016/B978-0-12-409548-9.095804>

Buschmann AH, VA Riquelme, M Hernández-González, CD Varela, JE Jiménez, LA Henríquez, PA Vergara, R Guíñez \& L Filún. 2006. A review of the impacts of salmon farming on marine coastal ecosystems in the southeast Pacific. ICES Journal of Marine Science 63: 1338-1345.

Buschmann AH, A Tomova, A López, MA Maldonado \& LA Henríquez. 2012. Salmon aquaculture and antimicrobial resistance in the marine environment. PLoS ONE 7(8): e42724. <doi: 10.1371/journal.pone.0042724>

Cabello FC. 2004. Antibióticos y acuicultura en Chile: consecuencias para la salud humana y animal. Revista Médica de Chile 132(8): 1001-1006.

Cabello FC. 2006. Heavy use of prophylactic antibiotics in aquaculture: a growing problem for human and animal health and for the environment. Environmental Microbiology 8(7): 1137-1144 
Cabello FC, HP Godfrey, A Tomova, L Ivanova, H Dölz, A Millanao \& AH Buschmann. 2013. Antimicrobial use in aquaculture re-examined: its relevance to antimicrobial resistance and to animal and human health. Environmental Microbiology 15(7): 1917-1942.

Coyne R, M Hiney, B O’Connor, D Cazabon \& P Smith. 1994. Concentration and persistence of oxytetracycline in sediments under a marine salmon farm. Aquaculture 123: $31-42$.

Dietze JE, EA Scribner, MT Meyer \& DW Kolpin. 2005. Occurrence of antibiotics in water from 13 fish hatcheries, 2001-2003: International Journal of Environmental Analytical Chemistry 85(15): 1141-1152.

Drummond AJ, B Ashton, S Burton, M Cheung, A Cooper, J Heled, R Moir, S Stones-Havas, S Sturrock \& T Thierer. 2011. Geneious v5.4. Biomatters, Auckland. http:/ /www.geneious.com/

Harms H, D Schlosser \& LY Wick. 2011. Untapped potential: exploiting fungi in bioremediation of hazardous chemicals. Nature Reviews Microbiology 9: 177-191.

Hektoen H, JA Berge, V Hormazábal \& M Yndestad. 1995. Persistence of antibacterial agents in marine sediments. Aquaculture 133: 175-184.

Heuer OE, H Kruse, K Grave, P Collignon \& I Karunasagar. 2009. Human health consequences of use of antimicrobial agents in aquaculture. Clinical Infectious Diseases 49: 1248-1253.

Kerry J, M Hiney, R Coyne, S NicGabhainn, D Gilroy, D Cazabon \& P Smith. 1995. Fish feed as a source of oxytetracycline-resistant bacteria in the sediments under fish farms. Aquaculture 131: 101-113.

Kong AYY, KM Scow, AL Córdova-Kreylos, WE Holmes \& J Six. 2011. Microbial community composition and carbon cycling within soil microenvironments of conventional, lowinput, and organic cropping systems. Soil Biology and Biochemistry 43:20-30.

Kristanti RA \& T Hadibarata. 2015. Biodegradation and identification of transformation products of fluorene by ascomycete fungi. Water Air and Soil Pollution 226(12): 406.

Kulshreshtha S, N Mathur \& P Bhatnagar. 2014. Mushroom as a product and their role in mycoremediation. AMB Express 4:29. <doi: 10.1186/s13568-014-0029-8>

Liu W, N Pan, W Chen, W Jiao \& M Wang. 2012. Effect of veterinary oxytetracycline on functional diversity of soil microbial community. Plant Soil Environment 58: 295-301.

Magan N, S Fragoeiro \& C Bastos. 2010. Environmental factors and bioremediation of xenobiotics using white rot fungi. Mycobiology 38: 238-248.

Maloney SE. 2001. Pesticide degradation. In: Gadd GM (ed). Fungi in bioremediation, pp. 188-223. Cambridge University Press, Cambridge.
Marshall BM \& SB Levy. 2011. Food animals and antimicrobials: impacts on human health. Clinical Microbiology Reviews 24: 718-733.

Miranda C \& R Zemelman. 2002. Bacterial resistance to oxytetracycline in Chilean salmon farming. Aquaculture 212: $31-47$.

Rudolph A, P Medina, C Urrutia \& R Ahumada. 2009. Ecotoxicological sediment evaluations in marine aquaculture areas of Chile. Environmental Monitoring and Assessment 155: 419-429.

Rudolph A, P Medina, R Ahumada \& V Novoa. 2011. Ecotoxicological quality of sediments in fiords in Southern Chile (44 - 46.5 ${ }^{\circ}$ LS). Revista de Biología Marina y Oceanografía 46: 79-84.

Samuelsen OB, V Torsvik \& A Etvik. 1992. Long-range changes in oxytetracycline concentration and bacterial resistance towards oxytetracycline in a fish farm sediment after medication. Science of the Total Environmental 114: 25-36.

Samuelsen OB, BT Lunestad, A Ervik \& S Fjelde. 1994. Stability of antimicrobial agents in artificial marine sediment studied under laboratory conditions. Aquaculture 126: 283-290.

Seo JS, YS Keum \& QX Li. 2009. Bacterial degradation of aromatic compounds. International Journal of Environmental Research and Public Health 6: 278-309

SERNAPESCA. 2014. Informe sobre uso de antimicrobianos en la salmonicultura nacional 2013, 19 pp. Unidad de Salud Animal, Ministerio de Economía, Fomento y Turismo, Servicio Nacional de Pesca y Acuicultura, Valparaíso. <http:// www.sernapesca.cl/presentaciones/Comunicaciones/ Informe_Sobre_Uso_de_Antimicrobianos_2015.pdf>

Smith P. 1996. Is sediment deposition the dominant fate of oxytetracycline used in marine salmonid farms: a review of available evidence. Aquaculture 146: 157-169.

Smith P \& OB Samuelsen. 1996. Estimates of the significance of out-washing of oxytetracycline from sediments under Atlantic salmon sea-cages. Aquaculture 144: 17-26.

Tedersoo L, T Suvi, T Jairus \& H Kõljalg. 2009. Forest microsite effects on community composition of ectomycorrhizal fungi on seedlings of Picea abies and Betula pendula. Environmental Microbiology 10: 1189-1201.

Vidaver A. 2002. Uses of antimicrobials in plant agriculture. Clinical Infectious Disease 34(3): 107-110.

Williams AJ, J Deck, JP Freeman, MP Chiarelli, MD Adjej, TM Heinze \& JB Sutherland. 2007. Biotransformation of flumequine by the fungus Cunninghamella elegans, Chemosphere 67: 240-243.

WingChing-Jones R. 2008. Residualidad de sustancias xenobióticas en el suelo empleadas en la producción pecuaria. Agronomía Mesoamericana 19(1): 99-114.

Recibido el 15 de marzo de 2016 y aceptado el 10 de septiembre de 2016

Editor: Claudia Bustos D. 